\title{
MULTIDIMENSIONAL MANAGEMENT OF STRESS-INDUCED INFERTILE WOMEN BY IVF
}

\author{
Dr.Kamath Madhusudhana \\ Associate Professor, \\ Division of Ayurveda, \\ Centre for Integrative Medicine and Research, \\ Manipal Academy of Higher Education, \\ Manipal-576 104 \\ Manipal-576 401
}

Article DOI: https://doi.org/10.36713/epra4311

\begin{abstract}
It was observed that psychogenic causes were plays an important role in conception ${ }^{1,2}$.WHO in 2006 clearly defines infertility as 'The inability of a couple to achieve conception or to bring a pregnancy to term after a year or more of regular, unprotected intercourse'.

It can be classified as either "primary" or "secondary". While Moshi says as infertility is the "absence of conception after 12 months of regular, unprotected intercourse'.Even the world is suffering from a population explosion still infertility is a global health issue!. The prevalence is most common in both sex still it mainly represents dominantly with the rate of 30-40\%.
\end{abstract}

\section{INTRODUCTION}

Varies difficulties experienced by infertile women identified by Yael Benyamini (2004). Among two hundred and forty-two women undergoing evaluation and treatment of fertility problems at the initial stages of treatment by administering infertility distress and well-being scales, a cross-sectional survey study was performed. It revealed twenty-two difficulties from infertility treatment. The experience of difficulties was related to more distress and lower well-being. The list of difficulties identified could assist health care providers and psychosocial counsellors in identifying misperceptions of difficulties that present in communication gaps between patients and providers and between patients and spouses were concluded by the study. Many couples feel helpless and loss of control over their body or their life plan. Infertility can be a major crisis because the important life goal of parenthood is threatened when diagnosed with infertility. It is more traumatic for women as motherhood is considered an essence of female role and identity. Education and support to cope with anxiety because the infertility treatment procedure is stressful, time-consuming and expensive.
Diagnosis of infertility
1. Loss of self-esteem,
2. 2.Socioeconomic status,
3. Marital and Homesickness
4. Emotional factors like Sexual pleasure.

\section{CAUSES OF INFERTILITY Physical Problems}

The study investigated by Leboeuf - Yde (2006)showed the relation between heights, body mass index, intellectual capacity, coping to stress and type of education with back problems and ill health. The associations between heights, body mass index, intellectual capacity, coping to stress and type of education with back problems and ill health were weak to moderate as shown in the result and a were strong association between coping and back problems and ill health. 


\section{Maternal Age}

The factors affecting the quality of life was identified by using a structured questionnaire ${ }^{3}$. The factors identified were mainly mood-related to women who were above 30 years and with polygamous marriages. The results highlighted continuing support for infertile women to improve their quality of life.

IVF success and greater use of donor oocytes have resulted in a higher percentage of births in women $\geq 40$ years of age ${ }^{4}$.

\section{Gender}

Health-related quality of life among 514 women and 514 men through the cross-sectional study was examined by ${ }^{5}$. It revealed that male patients had the better health-related quality of life compared to women who showed the poorer physical health-related quality of life; less educated younger women to be at risk of sub-optimal health-related quality of life. It suggests that infertility women should be provided with support to improve their wellbeing.

Retrospective questionnaires review done by Eileen Mary Conway's (2002) showed that there were gender differences in couples reported reactions to infertility. The majority of the women discussed that they were in more grief reactions than their partners and frequently said their responses to discovering an infertility problem were surprise and acceptance.

\section{Occupation}

The association of coping responses with infertility and occupational social class was analyzed by Christensen during 2005. 1169 women who were about to begin assisted reproduction treatment the data were collected. An adaptation of Lazarus and Folkman's Ways of Coping Questionnaire the coping measure was developed. Four categories were developed to measure: active-avoidance coping; active-confronting coping; passive-avoidance coping; meaning-based coping. These were later confirmed by factor analysis. Occupational social class was measured in a standardized way. The logistic regression analyses showed that women from lower social classes used significantly more active-confronting coping, more meaning-based coping. Due to the significant social differences in coping with infertility, the study suggests that the elements of coping may be learned from one's social network and the reference group.

\section{Stress Hormone}

The associations between urinary levels of neither stress hormones adrenaline, noradrenaline and cortisol during treatment among 168 women entering their first cycle of IVF / ICSI ${ }^{6}$. The result showed that women with successful treatment had lower concentrations of adrenaline at oocyte retrieval and lower concentrations of adrenaline and noradrenaline at ET, compared with unsuccessful women. It suggests that adrenal hormone may have a relationship between psychosocial stress and the outcome of IVF treatment.

The effect of chronic stress on the hypothalamicpituitary-adrenal cortex and the reproductive cycle ${ }^{7}$. There was a moderate association between stress during IVF treatment and outcome of the treatment the study on stress of infertility treatment concluded.

Samuel Wasser a study done in 1993 by the reproductive biologist, gives a clue about how stress may affect fertility. The battery of psychological tests to 38 women was given by him. He found that women whose infertility was caused by hormonal changes showed much higher levels of stress than those whose partners were found to be infertile or whose infertility problem may be due to the anatomical problems, like blocked fallopian tubes, etc. Infertility due to hormonal changes maybe because of the hypothalamus, a part of the brain that controls the flow and timing of reproductive hormones, which is sensitive to tension. The way the hypothalamus orchestrates the hormones may be affected by stress.

\section{WOMEN AND INFERTILITY STRESS Stress in Women}

Stress can involve a recent change or a daily pressure by Cleveland Clinic Foundation, (2009). It can happen to everyone and can be motivating and productive or negative and destructive. Emotional consequences of stress are tension and anxiety, as well as depression.

The survey conducted 8 involving 281 patients awaiting assisted reproduction treatment at five centres in three countries, and 289 population controls, were investigated whether the patients had experienced more negative emotional feelings and negative emotional impact during periods when they were attempting to conceive as compared with the controls and whether there was any difference in their well-being at the time of consultation. In the context of the burden of fertility problems, the study was performed. Questionnaires were used to survey the self-administration type. Women with fertility problems reported a higher prevalence of negative emotions than the controls. More changes were reported in inter partner relationships (either negative or positive). The negative effect of Sexuality was seen among them. One in four $(24.9 \%)$ of women had scores indicating depressive disorders as compared with only $6.8 \%$ of the controls and current well-being was even more affected in women with 
previous unsuccessful in-vitro fertilization (IVF)experience. The 'infertility' life event was perceived as severe by both patients and controls. Both before consultation and during diagnosis and treatment, women with fertility problems had a higher prevalence of reported negative psycho-emotional experiences than women without fertility problems.

\section{Diagnosis of Stress}

It is observed by 9, Among all couples undergoing the first cycle of IVF between 2002 and 2005 years fertility Problem Inventory was used. Couples who conceived during their first cycle of IVF had significantly higher measures of need for parenthood and loss of sexual enjoyment compared with couples who did not conceive was the resulting outcome. Couples who achieved ongoing pregnancies had higher scores on measures of a negative view of a child-free lifestyle need for parenthood, and total stress than those who did not.

Negative emotions such as anger, hostility, isolation, feeling blamed, feeling unsupported, feeling misunderstood, feeling that one's spouse is not equally committed to having children, worrying about a possible breakup of the relationship, and actions such as blaming of husband and wife are exhibited by women. It further conveyed that the attitudes, emotions, cognitions, and fantasies are variables that influence the course and outcome of a conflict.

\section{Prevalence of Stress in Infertility Women}

Depression in women is the leading cause of disease-related disability among women as stated by Nobel (2005). The lifetime prevalence of a major depressive disorder in women $(21.3 \%)$ is almost twice that in men $(12.7 \%)$ epidemiological studies have shown that. The predisposing factors were biological process for women to depression including genetically determined vulnerability, undue sensitivity to such hormonal fluctuations in biological systems may cause depression. Psychological events are contributing factors for women to develop depression such as role stress, victimization, sex-specific socialization, internalization coping style, and poor social support system. Depression can be also caused by reproductive cycles such as infertility, miscarriage, oral contraceptives, and hormone replacement treatment.

\section{The burden of the Disease}

It is 11 concluded that the risk factors of depression and anxiety in infertility would be being female sex, age over 30 years, lower level of education, lack of occupational activity diagnosed male infertility and infertility duration of 3-6 years.

A study conducted 12 to determined the prevalence, severity and predictability of psychiatric symptoms of infertile women and the effects of it on marital and sexual relationships.

It revealed that depression, anxiety and strength of psychological symptoms were significantly higher in the infertile group.

\section{DIMENSIONS OF THE INFERTILITY STRESS \\ Social Concern}

Infertility is the need for a woman to have a child remains basic. An individual woman's treatment in the community continues to be defined by Motherhood, her self-respect and her understanding of womanhood. It is a phase where an individual woman gets treatment well from society, gains self- respect and a better understanding of her womanhood.

It observed ${ }^{12}$ among women seeking infertility treatment, $83 \%$ reported a feeling of societal pressures to conceive. It also reports that women in the experimental group reported poor marital adjustment and quality of life when compared with controls.

\section{Sexual Concern}

The sexual dysfunction in 100 infertile couples 14 evaluated using a sexual function questionnaire (SFQ). The prevalence of female sexual dysfunction was high and low in arousal-sensation (80.2\%) and orgasm (22.8\%) domains, respectively.

A prospective study performed 15 to assess the frequency of intercourse before pregnancy and during the 1st trimester among sixteen infertility women and control women. Results showed that both groups experienced a significant decrease in intercourse frequency during the first trimester $(\mathrm{p}<.01)$.

The impact of stress on reproductive life and Self-esteem identified ${ }^{16}$ after a diagnosis of infertility many men and women reported feeling less masculine or feminine. Research has shown that women going through infertility rated themselves as having higher levels of depression and low self- esteem than women as going through cancer treatment.

\section{Marital Relationship}

Infertile couples' short-term emotional responses to their first IVF treatment (the women's and men's emotional reactions and their experiences of the marital relationship at different stages of the first treatment) and the outcome of the IVF treatment will be assessed ${ }^{16}$. The findings were reported as that women's and men's 
emotional reactions related to first IVF treatment were dependent on whether they achieved pregnancy or not.

\section{Family Stress}

It is observed that family stress among hospitalized women receiving infertility treatment with ovarian hyperstimulation syndrome.

\section{FACTORS THAT EMOTIONALLY INFLUENCE WOMEN DIAGNOSED AS INFERTILE \\ Uncertainty about the success of infertility treatment}

The reason for discontinuing from the IVF was investigated and the reason was the psychological burden in $26 \%$, poor prognosis in $25 \%$ and other reasons in $7 \%$. The study concluded an unexpectedly high percentage of couples discontinued the treatment before the three cycles. Majority of these discontinuations were due to psychological stress.

\section{Grief associated with infertility Diagnosis}

The psychological status of 120 Kuwaiti infertile women and age-matched samples of 125 healthy pregnant women were taken as a control group. The result revealed that infertile women exhibited significantly higher psychopathology in all HADS parameters in the form of tension, hostility, anxiety, depression, self-blame and suicidal ideation observed by Fido (2004).

\section{The feeling of Losing Control}

A study identifies the role of the treatment strategy applied, and potentially other factors that influence the decision of couples to discontinue treatment. The physical or psychological burden of treatment was the frequent cause of drop-out (28\%).

\section{Loss of Self-esteem}

A cross-sectional study ${ }^{19}$ with 68 women who were starting their first IVF treatment at the Leuven University Fertility Centre of the University of Leuven, Belgium. All were administered self-report measures assessing the self-criticism and dependency, negative life events, psychological wellbeing, relationship satisfaction, and relationship and sexual concerns. The result reveals that the high self-criticism was negatively associated with psychological wellbeing $(r=-0.63, P<$ $0.001)$ and relationship satisfaction $(r=-0.29, P<$ $0.05)$, and positively associated with relationship $(r=$ $0.39, P<0.01)$ and sexual $(r=0.37, P<0.01)$ concerns. High dependency was negatively associated with psychological wellbeing $(r=-0.30, P<0.05)$, not significantly associated with relationship satisfaction and relationship concerns, and positively related to sexual concerns $(r=0.31, P<0.05)$.
Infertility touches all aspects of a person's life. The feelings of self, their relationships and their life perspectives are affected quotes Hart (2002) "Couple experiencing ongoing stress for a long period may create guilt, anxiety, tension within the relationship, and feelings of depression and isolation".

\section{Financial Strain}

Article by Jenkins (2005) overviewed on the influence of ethnicity and socioeconomic status on the use of infertility services. The demographic variables of infertile women by race and economic factors.

\section{Sexual Pressure}

The stress could theoretically interfere with pregnancy through direct hormonal effects, or indirectly by impairing a couple's capacity to have effective sexual intercourse or to follow the complex instructions and sexual prescriptions involved in medical treatment. However, a major focus of mental health care for couples is to help them cope with the emotional impact of treatment Department of health (2004) reported.

\section{Pregnancy Outcome}

The study finding suggested that the relationship between psychological parameters and IVF success rates is more complex than commonly believed. The expression of negative emotions before starting IVF might not be always detrimental for outcomes ${ }^{20}$.

\section{YOGA AND ITS THERAPEUTIC USE FOR INFERTILE WOMEN DURING IVF TREATMENT \\ Yoga in Infertility Women}

Yoga is an adjunct to infertility treatment ${ }^{21}$. Yoga and meditation help women who are experiencing infertility. The practice of meditation and relaxation increases clarity of their mind, maintain healthy body chemistry and give the patience to undergo the rigours of treatments. Researchers in Hong Kong on "bodymind-spirit" form of group support eased anxiety levels in a group of women about to undergo IVF.

Eastern body - mind - spirit group intervention to help infertile women in the Chinese population develop by Chan, C. H. (2005) cope with the distress arising from treatment. The study adopted a biopsychosocial - spiritual health model. Results showed that and reflected that this approach could enhance the holistic health of these women.

\section{Physiology of yoga}

It is observed that the effect of meditation on Neurophysiological mechanisms inducing "meditative effect", "efferent attenuation", "sensory attenuation" and "cognitive restructuring" in varying degree of combinations to produce the effect during different types of meditations. 


\section{Yoga on Psychological Changes}

It is observed that 22 yogic breathing are a unique method for bringing balance in the autonomic nervous system and influencing psychological and stress-related disorders. The yogic technique enhances well being, mood, attention, mental focus, and stress tolerance. Acharya Anoop (2005) states that the benefit of Sukhasana posture is a vehicle for Pranayama, eliminates exhaustion and fatigue after strenuous work; Shavasana (pose of tranquillity) is the for relaxing the muscles of the body including the spine.

\section{Ayurveda and Infetiity}

Ayurveda I also one of the traditional medicinal system of India like Yoga. The detailed history of patients and various investigations of infertility has been done. On that basis it can be concluded that 99\% patients do not care about dincharya( daily regimen ), ratricharya ( Night regimen), ritucharya (seasonal regimen Sadvritta( Good conduct), and various health measures necessary for health. excessive exposure to radiation, vulgarity has shown on television, video, audio etc. and stressful fast life are the main causes. Ayurvedic detox therapy and oral medication providing comparatively more relief in cervical mucus amount, viscosity, fern and also on follicular growth.

\section{DISCUSSION}

Infertility has been one of the yet unsolved major complaints of womanhood. The system of modern medicine has discovered many of its etiological factors and provide measures for its management. According to the definition, infertility is diagnosed after one year of marriage. Therefore chronicity starts after one year and patients trying for the year till they get success, from different pathies and different medical centres. Maximum patients were at present instance educated up to Higher secondary which shows an increased tendency of education in Indian women and also shows their awareness towards their problem. Now a days integrative, muti dimensional, holistic method of treatments exist in the world, But we must take care of that theses various pathies may not cause, herb drug interaction, drug drug interaction, disease drug interaction, food drug interaction, the ultimate message this integrative therapy may not cause any harm to the patient.

\section{CONCLUSION}

In modern science, there is a good treatment for infertility due to anovulation, but in later stage it's side effects give worry. For its treatment clomiphene citrate is the drug of choice which is doing well in many women but still, it causes Polycystic Ovarian Disease (PCOD) and other side effects also. So I present paper more stress is given to the Diet regimen along with contemporary therapy.

\section{REFERENCES}

1. Rothman, D., Kaplan, AH , \& Nettles, E. ( 1962). Psychosomatic infertility. Ameri- can journal of Obstetrics \& Gynaecology, 83, 373-381

2. Burns, L. H., \& Covington, S. N. (1999). Psychology of infertility. Infertility Counseling. A Comprehensive Handbook for Clinicians. Parthenon Publishing, New York, London, 3-25.

3. Khayata, G. M., Rizk, D. E. E., Hasan, M. Y., Ghazal-Aswad, S., \& Asaad, M. A. N. (2003). Factors influencing the quality of life of infertile women in United Arab Emirates. International Journal of Gynecology \& Obstetrics, 80(2), 183188.

4. Paredes, R., Gutiérrez, J., Gutierrez, S., Allison, L., Puchi, M., Imschenetzky, M., \& Montecino, M. (2002). Interaction of the $1 \alpha, 25$-dihydroxyvitamin D3 receptor at the distal promoter region of the bone-specific osteocalcin gene requires nucleosomal remodelling. Biochemical Journal, 363(3), 667-676.

5. Hao, Y., Chun, A., Cheung, K., Rashidi, B., \& Yang, $X$. (2008). Tumor suppressor LATS1 is a negative regulator of oncogene YAP. Journal of Biological Chemistry, 283(9), 5496-5509.

6. Verhaak, C. M., Smeenk, J. M. J., Van Minnen, A., Kremer, J. A. M., \& Kraaimaat, F. W. (2005). A longitudinal, prospective study on emotional adjustment before, during and after consecutive fertility treatment cycles. Human reproduction, 20(8), 2253-2260.

7. Wilson, D., \& Sperber, D. (2002). Relevance theory.

8. den Tonkelaar, I., \& Oddens, B. J. (2000). Determinants of long-term hormone replacement therapy and reasons for early discontinuation. Obstetrics \& Gynecology, 95(4), 507-512.

9. Cooper, J. O., Heron, T. E., \& Heward, W. L. (2007). Applied behavior analysis.

10. Sartorius, A., Mahlstedt, M. M., Vollmayr, B., Henn, F. A., \& Ende, G. (2007). Elevated spectroscopic glutamate/ $\gamma$-amino butyric acid in rats bred for learned helplessness. Neuroreport, 18(14), 1469 1473.

11. Drosdzol, A., \& Skrzypulec, V. (2009). Depression and anxiety among Polish infertile couples-an evaluative prevalence study. Journal of Psychosomatic Obstetrics \& Gynecology, 30(1), 1120.

12. Ozkan, M., \& Baysal, B. (2006). Emotional distress of infertile women in Turkey. Clinical and experimental obstetrics \& gynecology, 33(1), 44-46.

13. Khademi, A., Alleyassin, A., Amini, M., \& Ghaemi, M. (2008). Evaluation of sexual dysfunction prevalence in infertile couples. The journal of sexual medicine, 5(6), 1402-1410. 
14. Optale, G., Marin, S., Pastore, M., Nasta, A., \& Pianon, C. (2003). Male sexual dysfunctions and multimedia immersion therapy (followup). CyberPsychology \& Behavior, 6(3), 289-294.

15. Strauß, B. (2004). Fertilitätsstörungenpsychosomatisch orientierte Diagnostik und Therapie: Leitlinie und Quellentext. Schattauer Verlag.

16. Holter, H., Anderheim, L., Bergh, C., \& Möller, A. (2006). First IVF treatment-short-term impact on psychological well-being and the marital relationship. Human reproduction, 21(12), 32953302 .

17. Lee, Y. J. (2008). Subjective quality of life measurement in Taipei. Building and Environment, 43(7), 1205-1215.

18. Verberg, M. F., Eijkemans, M. J., Macklon, N. S., Heijnen, E. M., Fauser, B. C., \& Broekmans, F. J. (2008). Predictors of ongoing pregnancy after single-embryo transfer following mild ovarian stimulation for IVF. Fertility and Sterility, 89(5), 1159-1165.

19. Lowyck, B., Luyten, P., Demyttenaere, K., \& Corveleyn, J. (2008). The role of romantic attachment and self-criticism and dependency for the relationship satisfaction of community adults. Journal of Family Therapy, 30(1), 78-95.

20. Pereira, G., Bell, M. L., Belanger, K., \& De Klerk, N. (2014). Fine particulate matter and risk of preterm birth and pre-labour rupture of membranes in Perth, Western Australia 1997-2007: a longitudinal study. Environment international, 73, 143-149.

21. Khalsa, H. K. (2003). Yoga: an adjunct to infertility treatment. Sexuality, Reproduction and Menopause, 1(1), 46-51.

22. Middleton, D., \& Brown, S. D. (2005). The social psychology of experience: Studies in remembering and forgetting. Sage. 